\title{
GENERALIZED EIGENFUNCTIONS AND SPECTRAL THEORY FOR STRONGLY LOCAL DIRICHLET FORMS
}

\author{
DANIEL LENZ ${ }^{1}$, PETER STOLLMANN ${ }^{2}$, AND IVAN VESELIĆ ${ }^{3}$
}

\begin{abstract}
We present an introduction to the framework of strongly local Dirichlet forms and discuss connections between the existence of certain generalized eigenfunctions and spectral properties within this framework. The range of applications is illustrated by a list of examples.
\end{abstract}

\section{INTRODUCTION}

There is a long history to the study of connections between the spectrum of a selfadjoint differential operator and properties of generalized solutions to the associated eigenvalue equation. In this context, the following two 'meta theorems' have attracted particular attention:

- Positive generalized eigenfunctions exist for energies below the spectrum and the spectrum begins at the energy, where positive generalized eigenfunctions cease to exist.

- The spectrum is given by those energies, for which a (suitably) bounded generalized solution exists.

The first statement is sometimes discussed under the name of 'Allegretto Piepenbrink theorem'. The second statement is discussed under the heading of 'Shnol theorem'. Precise versions (and proofs) of these statements have been given in various contexts. It turns out that the framework of (strongly local) Dirichlet forms allows one to give a unified and structurally rather simple discussion of these two results. This has recently be shown in [45] (for the Allegretto Piepenbrink theorem) and in [23. for the Shnol type result, see the results on expansion in eigenfunctions in [22] as well. The mentioned framework includes a variety of operators among them Schrödinger operators, (uniform) elliptic operators on manifolds and (suitable) quantum graphs. Accordingly, the mentioned results have a rather broad applicability.

Our aim here is to discuss this approach to basic spectral theory via Dirichlet forms in a way that is accessible to the non-specialist. In this way, we will not only feature the Shnol Theorem and the Allegretto Piepenbrink theorems of [23, 45] but also hope to advertise the use of Dirichlet forms in spectral problems. For this reason we also conclude the paper with a discussion of various applications. The results in this paper are concerned with strongly local Dirichlet forms. A study of similar results for non-local Dirichlet forms (e.g. graphs) can be found in [34.

Date: June 30, 2009.

2000 Mathematics Subject Classification. 35P05, 81Q10.

Key words and phrases. Dirichlet forms, weak solutions, spectral properties. 
The organisation of this paper is as follows. We give a introduction into Dirichlet froms in Sections 1, 2 and 3. This introduction is aimed at a nonspecialist. We then discuss a version of Allegretto-Piepenbrink Theorem in Section 4 and results related to Shnol's Theorem in Section 5. These sections contain sketches of ideas and proofs. Finally, we discuss applications in Section 6

\section{Strongly local Dirichlet forms}

In this section we describe the set-up used throughout the paper. We refer to [35] as to the classical standard reference as well as [21, 28, 36, 47] for literature on Dirichlet forms. We treat real and complex function spaces at the same time and write $\mathbb{K}$ to denote either $\mathbb{R}$ or $\mathbb{C}$.

Throughout we will work with a locally compact, separable metric space $X$ endowed with a positive Radon measure $m$ with $\operatorname{supp} m=X$.

Dirichlet forms. The central object of our studies is a regular Dirichlet form $\mathcal{E}$ with domain $\mathcal{D}$ in $L^{2}(X)$ and the selfadjoint operator $H_{0}$ associated with $\mathcal{E}$. In order to precisely define these notions we recall the basic terminology of Dirichlet forms: Consider a dense subspace $\mathcal{D} \subset L^{2}(X, m)$ and a sesquilinear and non-negative map $\mathcal{E}: \mathcal{D} \times \mathcal{D} \rightarrow \mathbb{K}$ such that $\mathcal{D}$ is closed with respect to the energy norm $\|\cdot\|_{\mathcal{E}}$, given by

$$
\|u\|_{\mathcal{E}}^{2}=\mathcal{E}[u, u]+\|u\|_{L^{2}(X, m)}^{2},
$$

in which case one speaks of a closed form in $L^{2}(X, m)$. In the sequel we will write

$$
\mathcal{E}[u]:=\mathcal{E}[u, u] .
$$

The selfadjoint operator $H_{0}$ associated with $\mathcal{E}$ is then characterized by

$$
D\left(H_{0}\right) \subset \mathcal{D} \text { and } \mathcal{E}[f, v]=\left(H_{0} f \mid v\right) \quad\left(f \in D\left(H_{0}\right), v \in \mathcal{D}\right) .
$$

Such a closed form is said to be a Dirichlet form if $\mathcal{D}$ is stable under certain pointwise operations; more precisely, $T: \mathbb{K} \rightarrow \mathbb{K}$ is called a normal contraction if $T(0)=0$ and $|T(\xi)-T(\zeta)| \leq|\xi-\zeta|$ for any $\xi, \zeta \in \mathbb{K}$ and we require that for any $u \in \mathcal{D}$ also

$$
T \circ u \in \mathcal{D} \text { and } \mathcal{E}[T \circ u] \leq \mathcal{E}[u] .
$$

In the real case, this condition is often replaced by equivalent but formally weaker statement involving $u \vee 0$ and $u \wedge 1$, see [35], Thm. 1.4.1 and [47, Section I.4.

A Dirichlet form is called regular if $\mathcal{D} \cap C_{c}(X)$ is large enough so that it is dense both in $\left(\mathcal{D},\|\cdot\|_{\mathcal{E}}\right)$ and $\left(C_{c}(X),\|\cdot\|_{\infty}\right)$, where $C_{c}(X)$ denotes the space of continuous functions with compact support.

Examples. Here, we discuss some examples showing the wide range of applicability of Dirichlet forms.

The Laplacian on Euclidean space. The Laplacian in Euclidean space is the typical example to be kept in mind. It is given by

$$
H_{0}=-\Delta \text { on } L^{2}(\Omega), \quad \Omega \subset \mathbb{R}^{d} \text { open, }
$$


in which case

$$
\mathcal{D}=W_{0}^{1,2}(\Omega) \text { and } \mathcal{E}[u, v]=\int_{\Omega}(\nabla u \mid \nabla v) d x .
$$

Note that for differentiable contractions $T: \mathbb{R} \longrightarrow \mathbb{R}$ the chain rule easily gives the crucial Dirichlet form property for real valued functions $u$ as

$$
\mathcal{E}(T u)=\int_{\Omega}(\nabla T u, \nabla T u) d x=\int_{\Omega}\left|T^{\prime}(u(x))\right|^{2}(\nabla u, \nabla u) d x \leq \mathcal{E}(u)
$$

as $\left|T^{\prime}(z)\right| \leq 1$ for all $z \in \mathbb{C}$.

Uniform elliptic operators in Euclidean space: In the previous example we can allow for quite irregular coefficients of the differential operator. More precisely, let $\Omega \subset \mathbb{R}^{d}$ open and let $A$ be a measurable map from $\Omega$ into the symmetric $d \times d$ matrices. Assume that there exist $c, C>$ such that the eigenvalues of $A(x)$ lie in $[c, C]$ for all $x \in \Omega$. Then, the form $\mathcal{E}_{A}$ defined on $W_{0}^{1,2}(\Omega)$ by

$$
\mathcal{E}_{A}[u, v]=\int_{\Omega}(A(x) \nabla u \mid \nabla v) d x
$$

is a regular Dirichlet form.

Laplace Beltrami and unifom elliptic operators on manifolds: The previous example can easily be generalized to Laplace Beltrami operators on Riemannian manifolds: Let $M$ be a Riemannian manifold with metric tensor $g$ and exterior derivative $d$. Then, the form

$$
\mathcal{E}_{c}(u, v):=\int_{M}(d u, d v) d x
$$

defined for $u, v \in C_{c}^{\infty}(M)$ is closable. The closure is a Dirichlet form and its domain of definition is given by $W_{0}^{1,2}(M)$. The generator is the Laplace Beltrami operator. Again, we can allow for a measurable map $A$ from $M$ into the symmetric linear maps on the corresponding cotangent spaces with eigenvalues lying in some interval $[c, C]$ for $c, C>0$ and obtain the Dirichlet form

$$
\mathcal{E}_{A}(u, v):=\int_{M}(A d u, d v) d x
$$

defined on $W_{0}^{1,2}(M)$. These examples can be further generalized to allow for some subriemannian manifolds. We will not give details here.

Quantum graphs with Kirchhoff boundary conditions: This example has received attention in recent times. We refrain from giving details here but refer to the last section of the paper.

Capacity. The capacity is a set function that allows one to measure the size of sets in a way that is adapted to the form $\mathcal{E}$.

For $U \subset X, U$ open, we define

$$
\operatorname{cap}(U):=\inf \left\{\|v\|_{\mathcal{E}}^{2} \mid v \in \mathcal{D}, \chi_{U} \leq v\right\}
$$

where we set $(\inf \emptyset=\infty)$. For arbitrary $A \subset X$, we then set

$$
\operatorname{cap}(A):=\inf \{\operatorname{cap}(U) \mid A \subset U\}
$$


(see [35], p. 61f.). We say that a property holds quasi-everywhere, short q.e., if it holds outside a set of capacity 0 . A function $f: X \rightarrow \mathbb{K}$ is said to be quasi-continuous, q.c. for short, if, for any $\varepsilon>0$ there is an open set $U \subset X$ with $\operatorname{cap}(U) \leq \varepsilon$ so that the restriction of $f$ to $X \backslash U$ is continuous.

A fundamental result in the theory of Dirichlet forms says that every $u \in \mathcal{D}$ admits a q.c. representative $\tilde{u} \in u$ (recall that $u \in L^{2}(X, m)$ is an equivalence class of functions) and that two such q.c. representatives agree q.e. Moreover, for every Cauchy sequence $\left(u_{n}\right)$ in $\left(\mathcal{D},\|\cdot\|_{\mathcal{E}}\right)$ there is a subsequence $\left(u_{n_{k}}\right)$ such that the $\left(\tilde{u}_{n_{k}}\right)$ converge q.e. (see [35], p.64f).

Whenever we will write expressions containing pointwise evaluations of functions $u$ in the future, we will assume that a quasi continuous representative has been chosen.

Strong locality and the energy measure. $\mathcal{E}$ is called strongly local if

$$
\mathcal{E}[u, v]=0
$$

whenever $u$ is constant a.s. on the support of $v$.

Every strongly local, regular Dirichlet form $\mathcal{E}$ can be represented in the form

$$
\mathcal{E}[u, v]=\int_{X} d \Gamma(u, v)
$$

where $\Gamma$ is a nonnegative sesquilinear mapping from $\mathcal{D} \times \mathcal{D}$ to the set of $\mathbb{K}$-valued Radon measures on $X$. It is determined by

$$
\int_{X} \phi d \Gamma(u, u)=\mathcal{E}[u, \phi u]-\frac{1}{2} \mathcal{E}\left[u^{2}, \phi\right]
$$

for realvalued $u \in \mathcal{D}, \phi \in \mathcal{D} \cap C_{c}(X)$ and called energy measure; see also [21.

Obviously, all examples discussed in the preceeding subsection are strongly local. In the case of the Laplacian in Euclidean space, the measure $\Gamma$ is given by $(\nabla u \mid \nabla v) d x$ appearing above.

We discuss properties of the energy measure next (see e.g. [21, 35, 67]).

The energy measure inherits strong locality from $\mathcal{E}$ viz $\chi_{U} d \Gamma(\eta, u)=0$ holds for any open $U \in X$ and any $\eta, u \in \mathcal{D}$ with $\eta$ constant on $U$. This directly allows one to extend $\Gamma$ to $\mathcal{D}_{\text {loc }}$ defined as

$\left\{u \in L_{\text {loc }}^{2} \mid\right.$ for all compact $K \subset X$ there is $\phi \in \mathcal{D}$ s.t. $\phi=u m$-a.e. on $\left.K\right\}$, We will denote this extension by $\Gamma$ again. This extension is strongly local again i.e. satisfies

$$
\chi_{U} d \Gamma(\eta, u)=0
$$

for any open $U \in X$ and any $\eta, u \in \mathcal{D}_{\text {loc }}$ with $\eta$ constant on $U$. The set $\mathcal{D}$ is then given as the set of all $u \in \mathcal{D}_{\text {loc }}$ with $\int 1 d \Gamma(u)<\infty$. The energy measure satisfies the Leibniz rule,

$$
d \Gamma(u \cdot v, w)=u d \Gamma(v, w)+v d \Gamma(u, w),
$$

for all $u, v \in \mathcal{D}_{\text {loc }} \cap L_{\text {loc }}^{\infty}(X)$. (In fact strong locality is of $\mathcal{E}$ is equivalent to the validity of the Leibniz rule for functions in $\mathcal{D} \cap L_{\text {loc }}^{\infty}$ ) The energy measure also satisfies the chain rule

$$
d \Gamma(\eta(u), w)=\eta^{\prime}(u) d \Gamma(u, w)
$$


whenever $u, w \in \mathcal{D}_{\text {loc }} \cap L_{\text {loc }}^{\infty}$ are real valued and $\eta$ is continuously differentiable.

We write $d \Gamma(u):=d \Gamma(u, u)$ and note that the energy measure satisfies the Cauchy-Schwarz inequality:

$$
\begin{aligned}
\int_{X}|f g| d|\Gamma(u, v)| & \leq\left(\int_{X}|f|^{2} d \Gamma(u)\right)^{\frac{1}{2}}\left(\int_{X}|g|^{2} d \Gamma(v)\right)^{\frac{1}{2}} \\
& \leq \frac{1}{2} \int_{X}|f|^{2} d \Gamma(u)+\frac{1}{2} \int_{X}|g|^{2} d \Gamma(v)
\end{aligned}
$$

for all $u, v \in \mathcal{D}_{\text {loc }}$ and $f, g: X \longrightarrow \mathbb{C}$ measurable.

Due to Leibniz rule the sets $\mathcal{D}$ and $\mathcal{D}_{\text {loc }}$ resp. have certain closedness properties under multiplication. This is an interesting feature and we discuss it next.

It is not hard to see that any function in $u \in \mathcal{D}_{\text {loc }}$ with compact support belongs in fact to $\mathcal{D}$ (as $\int d \Gamma(u)=\int_{\text {supp } u} d \Gamma(u)<\infty$ ). More generally, localized versions of functions from $\mathcal{D}_{\text {loc }}$ belong to $\mathcal{D}$. More precisely, the following holds [45].

Lemma 1.1. (a) Let $\Psi \in \mathcal{D}_{l o c} \cap L_{l o c}^{\infty}(X)$ and $\varphi \in \mathcal{D} \cap L_{c}^{\infty}(X)$ be given. Then, $\varphi \Psi$ belongs to $\mathcal{D}$.

(b) Let $\Psi \in \mathcal{D}_{\text {loc }}$ and $\varphi \in \mathcal{D} \cap L_{c}^{\infty}(X)$ be such that $d \Gamma(\varphi) \leq C \cdot d m$. Then, $\varphi \Psi$ belongs to $\mathcal{D}$.

Part (a) of the lemma gives in particular that $\mathcal{D} \cap C_{c}(X)=\mathcal{D}_{\text {loc }} \cap C_{c}(X)$ and $\mathcal{D} \cap L_{c}^{\infty}(X)=\mathcal{D}_{\text {loc }} \cap L_{c}^{\infty}(X)$ are closed under multiplication.

In order to introduce weak solutions on open subsets $U$ of $X$, we extend $\mathcal{E}$ to $\mathcal{D}_{\text {loc }}(U) \times \mathcal{D}_{c}(U)$ : where,

$\mathcal{D}_{\text {loc }}(U):=\left\{u \in L_{\text {loc }}^{2}(U) \mid \forall\right.$ compact $K \subset U \exists \phi \in \mathcal{D}$ s.t. $\phi=u m$-a.e. on $\left.K\right\}$ $\mathcal{D}_{c}(U):=\{\varphi \in \mathcal{D} \mid \operatorname{supp} \varphi$ compact in $U\}$.

For $u \in \mathcal{D}_{\text {loc }}(U), \varphi \in \mathcal{D}_{c}(U)$ we define

$$
\mathcal{E}[u, \varphi]:=\mathcal{E}[\eta u, \varphi] .
$$

Here, $\eta \in \mathcal{D} \cap C_{c}(U)$ is arbitrary with constant value 1 on the support of $\varphi$. This makes sense as the RHS does not depend on the particular choice of $\eta$ by strong locality.

Obviously, also $\Gamma$ extends to a mapping $\Gamma: \mathcal{D}_{\text {loc }}(U) \times \mathcal{D}_{\text {loc }}(U) \rightarrow \mathcal{M}_{R}(U)$.

The intrinsic metric, strict locality and cut-off functions. Using the energy measure one can define the intrinsic metric

$$
\rho: X \times X \longrightarrow[0, \infty]
$$

by

$$
\rho(x, y)=\sup \left\{|u(x)-u(y)| \mid u \in \mathcal{D}_{\text {loc }} \cap C(X) \text { and } d \Gamma(u) \leq d m\right\}
$$

where the latter condition signifies that $\Gamma(u)$ is absolutely continuous with respect to $m$ and the Radon-Nikodym derivative is bounded by 1 on $X$. Despite its name, in general, $\rho$ need not be a metric. However, it is a pseudo metric viz it is symmetric, satisfies $\rho(x, x)=0$ for all $x \in X$ and satisfies the triangle inequality. 
We say that $\mathcal{E}$ is strictly local if $\rho$ is a metric that induces the original topology on $X$.

Note that strict locality implies that $X$ is connected, since otherwise points in $x, y$ in different connected components would give $\rho(x, y)=\infty$, as characteristic functions of connected components are continuous and have vanishing energy measure.

We denote the intrinsic balls by

$$
B(x, r):=\{y \in X \mid \rho(x, y) \leq r\} .
$$

An important consequence of strict locality is that the distance function $\rho_{x}(\cdot):=\rho(x, \cdot)$ itself is a function in $\mathcal{D}_{\text {loc }}$ with $d \Gamma\left(\rho_{x}\right) \leq d m$, see [67]. This easily extends to the fact that for every closed $E \subset X$ the function $\rho_{E}(x):=\inf \{\rho(x, y) \mid y \in E\}$ enjoys the same properties (see the Appendix of [23]). This has a very important consequence. Whenever $\zeta: \mathbb{R} \longrightarrow \mathbb{R}$ is continuously differentiable, and $\eta:=\zeta \circ \rho_{E}$, then $\eta$ belongs to $\mathcal{D}_{\text {loc }}$ and satisfies

$$
d \Gamma(\eta)=\left(\zeta^{\prime} \circ \rho_{E}\right)^{2} d \Gamma\left(\rho_{E}\right) \leq\left(\zeta^{\prime} \circ \rho_{E}\right)^{2} d m .
$$

For this reason a lot of good cut-off functions are around in our context. More explicitly we note the following lemma (Lemma 1.3 in [45], see [23] as well).

Lemma 1.2. For any compact $K$ in $X$ there exists $a \varphi \in C_{c}(X) \cap \mathcal{D}$ with $\varphi \equiv 1$ on $K, \varphi \geq 0$ and $d \Gamma(\varphi) \leq C d m$ for some $C>0$. If $L$ is another compact set containing $K$ in its interior, then $\varphi$ can be chosen to have support in $L$.

Irreducibility. We will now discuss a notion that will be crucial in the proof of the existence of positive weak solutions below the spectrum. In what follows, $\mathfrak{h}$ will denote a densely defined, closed semibounded form in $L^{2}(X)$ with domain $D(\mathfrak{h})$ and positivity preserving semigroup $\left(T_{t} ; t \geq 0\right)$. We denote by $H$ the associated operator. Actually, the cases of interest in this paper are the situation that $\mathfrak{h}=\mathcal{E}$ is a Dirichlet form as discussed above, or a measure perturbation thereof $\mathfrak{h}=\mathcal{E}+\nu$. Here it is assumed that the positive and negative part of the measure $\nu$ obey $\nu_{+} \in \mathcal{M}_{R, 0}, \nu_{-} \in \mathcal{M}_{R, 1}$, where the classes $\mathcal{M}_{R, 0}, \mathcal{M}_{R, 1}$ are discussed in the next section.

We say that $\mathfrak{h}$ is reducible, if there is a measurable set $M \subset X$ such that $M$ and its complement $M^{c}$ are nontrivial (have positive measure) and $L^{2}(M)$ is a reducing subspace for $M$, i.e., $\mathbb{1}_{M} D(\mathfrak{h}) \subset D(\mathfrak{h}), \mathfrak{h}$ restricted to $\mathbb{1}_{M} D(\mathfrak{h})$ is a closed form and $\mathcal{E}(u, v)=\mathcal{E}\left(u \mathbb{1}_{M}, v \mathbb{1}_{M}\right)+\mathcal{E}\left(u \mathbb{1}_{M^{c}}, v \mathbb{1}_{M^{c}}\right)$ for all $u, v$. If there is no such decomposition of $\mathfrak{h}$, the latter form is called irreducible. Note that reducibility can be rephrased in terms of the semigroup and the resolvent:

Theorem 1.3. Let $\mathfrak{h}$ be as above. Then the following conditions are equivalent:

(i) $\mathfrak{h}$ is irreducible.

(ii) $T_{t}$ is positivity improving, for every $t>0$, i.e. $f \geq 0$ and $f \neq 0$ implies that $T_{t} f>0$ a.e.

(iii) $(H+E)^{-1}$ is positivity improving for every $E<\inf \sigma(H)$. 
We refer to [56], XIII.12 and a forthcoming paper [46] for details.

It is quite easy to see that a disconnected space easily leads to reducible forms. The converse is not exactly right, but there are recent results that go far in this direction and characterize irreducibility, cf [31].

Assumptions. For the convenience of the reader we gather in this section assumptions and notation used in the sequel.

We will exclusively deal with regular, strongly local Dirichlet forms $\mathcal{E}$. The corresponding energy measure is denoted by $\Gamma$. The associated intrinsic metric is denoted by $\rho$.

We always chose quasi continuous representatives for elements of $\mathcal{D}_{\text {loc }}$.

The strongly local Dirichlet form $\mathcal{E}$ is strictly local if $\rho$ is a metric that induces the original topology on $X$. This condition can be slightly weakened. It suffices to assume that the pseudometric $\rho$ induces the original topology on $X$ (as for a given $x \in X$ one can then always restrict attention to the set of $y$ with $\rho(x, y)<\infty)$.

Later we will also encounter a growth assumption on the intrinsic metric.

(G) All intrinsic balls have finite volume with subexponential growth:

$$
e^{-\alpha \cdot R} m(B(x, R)) \rightarrow 0 \text { as } R \rightarrow \infty \text { for all } x \in X, \alpha>0 .
$$

Finally, we note that $\mathcal{E}$ is called ultracontractive if for each $t>0$ the semigroup $e^{-t H_{0}}$ gives a map from $L^{2}(X)$ to $L^{\infty}(X)$.

\section{Measure perturbations}

We will be dealing with Schrödinger type operators, i.e., perturbations $H=H_{0}+V$, where $H_{0}$ is associated to a strictly local Dirichlet form and the function $V$ is a suitable potential. In fact, we can even include measures as potentials. Here, we follow the approach from [64, 65]. Measure perturbations have been regarded by a number of authors in different contexts, see e.g. [11, 37, 67] and the references there.

We denote by $\mathcal{M}_{R}(U)$ the signed Radon measures on the open subset $U$ of $X$ and by $\mathcal{M}_{R, 0}(U)$ the subset of measures $\nu$ that do not charge sets of capacity 0 , i.e., those measures with $\nu(B)=0$ for every Borel set $B$ with $\operatorname{cap}(B)=0$. In case that $\nu=\nu_{+}-\nu_{-} \in \mathcal{M}_{R, 0}(X)$ we can define

$$
\nu[u, v]=\int_{X} \tilde{u} \bar{v} d \nu \text { for } u, v \in \mathcal{D} \text { with } \tilde{u}, \tilde{v} \in L^{2}\left(X, \nu_{+}+\nu_{-}\right) .
$$

Of course, a special instance of such measures is given by $\nu=V d m$ whenver $V$ belongs to $L_{\text {loc }}^{1}(X)$.

We have to rely upon more restrictive assumptions concerning the negative part $\nu_{-}$of our measure perturbation. We write $\mathcal{M}_{R, 1}$ for those measures $\nu \in \mathcal{M}_{R}(X)$ that are $\mathcal{E}$-bounded with bound less than one; i.e. measures $\nu$ for which there is a $\kappa<1$ and a $c_{\kappa} \geq 0$ such that

$$
\nu[u, u] \leq \kappa \mathcal{E}[u]+c_{\kappa}\|u\|^{2} .
$$

The set $\mathcal{M}_{R, 1}$ can easily be seen to be a subset of $\mathcal{M}_{R, 0}$.

By the KLMN theorem (see [55], p. 167), the sum $\mathcal{E}+\nu$ given by $D(\mathcal{E}+$ $\nu)=\left\{u \in \mathcal{D} \mid \tilde{u} \in L^{2}\left(X, \nu_{+}\right)\right\}$is closed and densely defined (in fact $\mathcal{D} \cap$ 
$\left.C_{c}(X) \subset D(\mathcal{E}+\nu)\right)$ for $\nu$ with $\nu_{+} \in \mathcal{M}_{R, 0}, \nu_{-} \in \mathcal{M}_{R, 1}$. We denote the associated selfadjoint operator by $H_{0}+\nu$. Note that $\mathcal{D} \cap L_{c}^{\infty}(X) \subset D(\mathcal{E}+\nu)$.

An important subclass of $\mathcal{M}_{R, 0}$ with very nice properties of the associated operators is the Kato class and the extended Kato class. In the present framework it can be defined in the following way: For $\mu \in \mathcal{M}_{0}$ and $\alpha>0$ we set

$$
\begin{gathered}
\Phi(\mu, \alpha): C_{c}(X)_{+} \rightarrow[0, \infty], \\
\Phi(\mu, \alpha) \varphi:=\int_{X}\left(\left(H_{0}+\alpha\right)^{-1} \varphi\right)^{\sim} d \mu .
\end{gathered}
$$

The extended Kato class is defined as

$$
\hat{\mathcal{S}}_{K}:=\left\{\mu \in \mathcal{M}_{0} \mid \exists \alpha>0: \Phi(\mu, \alpha) \in L^{1}(X, m)^{\prime}\right\}
$$

and, for $\mu \in \hat{\mathcal{S}}_{K}$ and $\alpha>0$,

$$
c_{\alpha}(\mu):=\|\Phi(\mu, \alpha)\|_{L^{\infty}(X, m)}\left(=\|\Phi(\mu, \alpha)\|_{L^{1}(X, m)^{\prime}}\right), c_{K a t o}(\mu):=\inf _{\alpha>0} c_{\alpha}(\mu) .
$$

The Kato class is originally defined via the fundamental solution of the Laplace equation in the classical case. In our setting it consists of those measures $\mu$ with $c_{\text {Kato }}(\mu)=0$.

As done in various papers, one can even allow for more singular measures, a direction we are not going to explore here.

As already discussed our measure perturbations preserve closability of the form. They preserve further properties. In fact, regularity is preserved in our context as well.

Theorem 2.1 (45]). Let $(\mathcal{E}, \mathcal{D})$ be a strongly local, regular Dirichlet form. Let $\nu$ with $\nu_{+} \in \mathcal{M}_{R, 0}, \nu_{-} \in \mathcal{M}_{R, 1}$ be given. Then, the perturbed form $(\mathcal{E}+\nu, D(\mathcal{E}+\nu))$ is regular as well.

Measure perturbations also preserve irreducibility, as can be seen from the following result.

Theorem 2.2 ([46]). Let $(\mathcal{E}, \mathcal{D})$ be a strictly local, regular, irreducible Dirichlet form. Let $\nu$ with $\nu_{+} \in \mathcal{M}_{R, 0}, \nu_{-} \in \mathcal{M}_{R, 1}$ be given. Then, the perturbed form $(\mathcal{E}+\nu, D(\mathcal{E}+\nu))$ is irreducible as well.

\section{WEAK SOLUTIONS}

Our main aim is to relate properties of weak solutions or generalized eigenfunctions to spectral properties of $H_{0}+\mu$. The necessary notation concerning weak solutions is introduced in this section. Throughout this section we consider a strongly local, regular Dirichlet form, $(\mathcal{E}, \mathcal{D})$ on $X$ and denote by $\Gamma: \mathcal{D}_{\text {loc }} \times \mathcal{D}_{\text {loc }} \rightarrow \mathcal{M}(X)$ the associated energy measure. We will be concerned with weak solutions $\Phi$ of the equation

$$
\left(H_{0}+V\right) \Phi=\lambda \cdot \Phi,
$$

where $H_{0}$ is the operator associated with $\mathcal{E}$ and $V$ is a realvalued, locally integrable potential. In fact, we will consider a somewhat more general framework, allowing for measures instead of functions, as presented in the previous section. Moreover, we stress the fact that (3.1) is formal in the sense that $\Phi$ is not assumed to be in the operator domain of neither $H_{0}$ nor $V$. Here are the details. 
Recall that we could extend $\Gamma$ to a measure valued function on $U$. In the same way, we can extend $\nu[\cdot, \cdot]$, using that every $u \in \mathcal{D}_{\text {loc }}(U)$ admits a quasi continuous version $\tilde{u}$.

Definition 3.1. Let $U \subset X$ be open and $\nu \in \mathcal{M}_{R, 0}(U)$ be a signed Radon measure on $U$ that charges no set of capacity zero. Let $\lambda \in \mathbb{R}$ and $\Phi \in$ $L_{l o c}^{2}(U)$. We say that $\Phi$ is a weak solution of $\left(H_{0}+\nu\right) \Phi=\lambda \cdot \Phi$ in $U$ if:

(i) $\Phi \in \mathcal{D}_{l o c}(U)$,

(ii) $\tilde{\Phi} d \nu \in \mathcal{M}_{R}(U)$,

(iii) $\forall \varphi \in \mathcal{D} \cap C_{c}(U)$,

$$
\mathcal{E}[\Phi, \varphi]+\int_{U} \varphi \tilde{\Phi} d \nu=\lambda \cdot(\Phi \mid \varphi) .
$$

If $V \in L_{l o c}^{1}(U)$ we say that $\Phi$ is a weak solution of $\left(H_{0}+V\right) \Phi=\lambda \cdot \Phi$ in $U$ if it is a weak solution of $\left(H_{0}+\nu\right) \Phi=\lambda \cdot \Phi$ for $\nu=V d m$.

Next, we briefly discuss these assumptions.

Remark 3.2. (1) If $\nu=V d m$ and $V \in L_{l o c}^{2}(U)$, then property (ii) of the Definition above is satisfied.

(2) If $\Phi \in L_{l o c}^{\infty}(U)$ and $\nu \in \mathcal{M}_{R}(U)$ then (ii) of the Definition above is satisfied.

(3) If $\nu \in \mathcal{M}_{R}(U)$ satisfies (ii) above then $\nu-E d m \in \mathcal{M}_{R}(U)$ satisfies (ii) as well and any weak solution of $\left(H_{0}+\nu\right) \Phi=\lambda \cdot \Phi$ in $U$ is a weak solution of $\left(H_{0}+\nu-E d m\right) \Phi=0$ in $U$. Thus it suffices to consider the case $\lambda=0$.

(4) By regularity we can replace (iii) by $\mathcal{E}[\Phi, \varphi]+\int_{U} \varphi \tilde{\Phi} d \nu=\lambda \cdot(\Phi \mid \varphi)$ for all $\varphi \in \mathcal{D}_{\text {loc }} \cap L_{c}^{\infty}(U)$ (see [45] for details).

\section{Positive weak solutions and the infimum of the SPeCtrum}

Throughout this section we consider a strongly local, regular Dirichlet form, $(\mathcal{E}, \mathcal{D})$ on $X$ and denote by $\Gamma: \mathcal{D}_{\text {loc }} \times \mathcal{D}_{\text {loc }} \rightarrow \mathcal{M}(X)$ the associated energy measure. The results discussed in this section are taken from [45] to which we refer for further details and proofs.

Ground state transform and consequences. We start with a theorem giving the so called ground state transform in our general setting.

Theorem 4.1. Let $(\mathcal{E}, \mathcal{D})$ be a regular, strictly local Dirichlet form, $H_{0}$ be the associated operator and $\nu$ a measure with $\nu_{+} \in \mathcal{M}_{R, 0}, \nu_{-} \in \mathcal{M}_{R, 1}$. Suppose that $\Phi$ is a weak solution of $\left(H_{0}+\nu\right) \Phi=\lambda \cdot \Phi$ in $X$ with $\Phi>0$ $m$-a.e. and $\Phi, \Phi^{-1} \in L_{l o c}^{\infty}(X)$. Then, for all $\varphi, \psi \in D(\mathcal{E}+\nu)$, the products $\varphi \Phi^{-1}, \psi \Phi^{-1}$ belong to $\mathcal{D}_{\text {loc }}$ and the formula

$$
\mathcal{E}[\varphi, \psi]+\nu[\varphi, \psi]=\int_{X} \Phi^{2} d \Gamma\left(\varphi \Phi^{-1}, \psi \Phi^{-1}\right)+\lambda \cdot(\varphi \mid \psi)
$$

holds.

The proof of the theorem proceeds essentially in two steps. In the first step a local version of the theorem is proven for 'smooth' $u, v$. In the second step this local version is then extended to the whole space. Note also that the conditions on $\Phi$ in the theorem imply that $\Phi^{-1}$ is in $\mathcal{D}_{\text {loc. }}$. As the 
local version may be of independent interest and has a very simple proof we include statement and proof next.

Theorem 4.2. Let $(\mathcal{E}, \mathcal{D})$ be a regular, strictly local Dirichlet form, $H_{0}$ be the associated operator and $\nu \in \mathcal{M}_{R, 0}(U)$. Suppose that $\Phi$ is a weak solution of $\left(H_{0}+\nu\right) \Phi=\lambda \cdot \Phi$ in $U$ with $\Phi>0$ m-a.e. and $\Phi, \Phi^{-1} \in L_{l o c}^{\infty}(U)$. Then, for all $\varphi, \psi \in \mathcal{D} \cap L_{c}^{\infty}(U)$ :

$$
\mathcal{E}[\varphi, \psi]+\nu[\varphi, \psi]=\int_{U} \Phi^{2} d \Gamma\left(\varphi \Phi^{-1}, \psi \Phi^{-1}\right)+\lambda \cdot(\varphi \mid \psi) .
$$

Proof. For the proof we may assume $\lambda=0$ without restriction. Without loss of generality we may also assume that $\varphi$ and $\psi$ are real valued functions. We now evaluate the RHS of the above equation, using the following identity. The Leibniz rule implies that for arbitrary $w \in \mathcal{D}_{\text {loc }}(U)$ :

$$
0=d \Gamma(w, 1)=d \Gamma\left(w, \Phi \Phi^{-1}\right)=\Phi^{-1} d \Gamma(w, \Phi)+\Phi d \Gamma\left(w, \Phi^{-1}\right)
$$

Therefore, for $\varphi, \psi \in \mathcal{D} \cap C_{c}(X)$ :

$$
\begin{aligned}
\int_{X} \Phi^{2} d \Gamma\left(\varphi \Phi^{-1}, \psi \Phi^{-1}\right)= & \int_{X} \Phi d \Gamma\left(\varphi, \psi \Phi^{-1}\right)+\int_{X} \Phi^{2} \varphi d \Gamma\left(\Phi^{-1}, \psi \Phi^{-1}\right) \\
\text { (by symmetry) }= & \int_{X} d \Gamma(\varphi, \psi)+\int_{X} \Phi \psi d \Gamma\left(\varphi, \Phi^{-1}\right) \\
& +\int_{X} \Phi^{2} \varphi d \Gamma\left(\psi \Phi^{-1}, \Phi^{-1}\right) \\
= & \mathcal{E}[\varphi, \psi]+\int_{X} \Phi^{2} d \Gamma\left(\varphi \psi \Phi^{-1}, \Phi^{-1}\right) \\
(\text { by }(\star))= & \mathcal{E}[\varphi, \psi]-\int_{X} d \Gamma\left(\varphi \psi \Phi^{-1}, \Phi\right) \\
= & \mathcal{E}[\varphi, \psi]-\mathcal{E}\left[\varphi \psi \Phi^{-1}, \Phi\right] .
\end{aligned}
$$

As $\Phi$ is a weak solution we can now use part (4) of the previous remark to continue the computation by

$$
\begin{aligned}
\ldots & =\mathcal{E}[\varphi, \psi]-\left(-\nu\left[\varphi \psi \Phi^{-1}, \Phi\right]\right) \\
& =\mathcal{E}[\varphi, \psi]+\nu[\varphi, \psi] .
\end{aligned}
$$

This finishes the proof.

The ideas behind our proof allow for some further generalizations. This is shortly indicated in the following remark. textbfRemark.

(1) This proof actually shows the following statement: Assume that there is a weak supersolution $\Phi$ of $\left(H_{0}+\nu\right) \Phi=\lambda \cdot \Phi$ on $X$ with $\Phi>0 m$-a.e. and $\Phi, \Phi^{-1} \in L_{\text {loc }}^{\infty}(X)$. Then $\mathcal{E}+\nu \geq \lambda$.

(2) We can allow for complex measures $\nu$ without problems. In the context of PT-symmetric operators there is recent interest in this type of Schrödinger operators, see 15 .

(3) Instead of measures also certain distributions could be included. Cf [38] for such singular perturbations.

We explicitely note the following immediate consequence of (both) of the theorems of this section. 
Corollary 4.3. Let $(\mathcal{E}, \mathcal{D})$ be a regular, strictly local Dirichlet form, $H_{0}$ be the associated operator and $\nu$ a measure on $X$ with $\nu_{+} \in \mathcal{M}_{R, 0}, \nu_{-} \in \mathcal{M}_{R, 1}$. Suppose that $\Phi$ is a weak solution of $\left(H_{0}+\nu\right) \Phi=\lambda \cdot \Phi$ in $X$ with $\Phi>0$ $m$-a.e. and $\Phi, \Phi^{-1} \in L_{l o c}^{\infty}(X)$. Then, $H_{0}+\nu \geq \lambda$.

Harnack principles and existence of positive solutions below the spectrum. The previous subsection shows that $H_{0}+\nu \geq \lambda$ whenever $\mathcal{E}+\nu$ is closable and admits a positive weak solution of $\left(H_{0}+\nu\right) \Phi=\lambda \Phi$. In this subsection discuss the converse under suitable conditions. A key property is related to the celebrated Harnack inequality.

Definition 4.4. (1) We say that $H_{0}+\nu$ satisfies a Harnack inequality for $\lambda \in \mathbb{R}$ if, for every relatively compact, connected open $X_{0} \subset X$ there is a constant $C$ such that all positive weak solutions $\Phi$ of $\left(H_{0}+\nu\right) \Phi=\lambda \Phi$ on $X_{0}$ are locally bounded and satisfy

$$
\operatorname{esssup}_{B(x, r)} u \leq C \operatorname{essinf}_{B(x, r)} u,
$$

for every $B(x, r) \subset X_{0}$ where esssup and essinf denote the essential supremum and infimum.

(2) We say that $H_{0}+\nu$ satisfies the Harnack principle for $\lambda \in \mathbb{R}$ if for every relatively compact, connected open subset $U$ of $X$ and every sequence $\left(\Phi_{n}\right)_{n \in \mathbb{N}}$ of nonnegative solutions of $\left(H_{0}+\nu\right) \Phi=\lambda \cdot \Phi$ in $U$ the following implication holds: If, for some measurable subset $A \subset U$ of positive measure

$$
\sup _{n \in \mathbb{N}}\left\|\Phi_{n} \mathbb{1}_{A}\right\|_{2}<\infty
$$

then, for all compact $K \subset U$ also

$$
\sup _{n \in \mathbb{N}}\left\|\Phi_{n} \mathbb{1}_{K}\right\|_{2}<\infty .
$$

(3) We say that $H_{0}+\nu$ satisfies the uniform Harnack principle if for every bounded intervall $I \subset \mathbb{R}$, every relatively compact, connected open subset $U$ of $X$ and every sequence $\left(\Phi_{n}\right)_{n \in \mathbb{N}}$ of nonnegative solutions of $\left(H_{0}+\nu\right) \Phi=\lambda_{n} \cdot \Phi$ in $U$ with $\lambda_{n} \in I$ the following implication holds: If, for some measurable subset $A \subset U$ of positive measure

$$
\sup _{n \in \mathbb{N}}\left\|\Phi_{n} \mathbb{1}_{A}\right\|_{2}<\infty
$$

then, for all compact $K \subset U$ also

$$
\sup _{n \in \mathbb{N}}\left\|\Phi_{n} \mathbb{1}_{K}\right\|_{2}<\infty .
$$

Note that validity of a Harnack principle implies that a nonnegative weak solution $\Phi$ must vanish identically if it vanishes on a set of positive measure (as $\Phi_{n}:=n \Phi$ has vanishing $L^{2}$ norm on the set of positive measure in question). Note also that validity of an Harnack inequality extends from balls to compact sets by a standard chain of balls argument. This easily shows that $H_{0}+\nu$ satisfies the Harnack principle for $\lambda \in \mathbb{R}$ if it obeys a Harnack inequality for $\lambda \in \mathbb{R}$. Therefore, many situations are known in which the Harnack principle is satisfied: 
For $\nu \equiv 0$ and $\lambda=0$ a Harnack inequality holds, whenever $\mathcal{E}$ satisfies a Poincaré and a volume doubling property; cf [20] and the discussion there. The most general results for $H_{0}=-\Delta$ in terms of the measures $\nu$ that are allowed seem to be found in [37. The uniformity of the estimates from [37] immediately gives that the uniform Harnack principle is satisfied for Kato class measures. Of the enormous list of papers on Harnack's inequality, let us also mention [8, 18, 19, 26, 37, 39, 41, 49, 57, 58, 69, 70]

Apart from the Harnack principle there is a second property that will be important in the proof of existence of positive general eigensolutions at energies below the spectrum.

Definition 4.5. The form $\mathcal{E}$ satisfies the local compactness property if $D_{0}(U):=\overline{D \cap C_{c}(U)}\left\|^{\|}\right\|_{\mathcal{E}}$ is compactly embedded in $L^{2}(X)$ for every relatively compact open $U \subset X$.

In case of the classical Dirichlet form the local compactness property follows from Rellich's Theorem on compactness of the embedding of Sobolev spaces in $L^{2}$.

It turns out that the situation is somewhat different depending on whether $X$ is compact or not. In both cases we will need the assumption of irreducibility in order to obtain solutions which are positive almost everywhere. This is clear as in the reducible case a nontrivial solution could still vanish on some 'components'.

We first get the case of compact $X$ out of our way.

Theorem 4.6. Let $(\mathcal{E}, \mathcal{D})$ be a regular, strictly local, irreducible Dirichlet form, $H_{0}$ be the associated operator and $\nu$ a measure with $\nu_{+} \in \mathcal{M}_{R, 0}, \nu_{-} \in$ $\mathcal{M}_{R, 1}$. Suppose that $X$ is compact and $\mathcal{E}$ satisfies the local compactness property. Then, $H_{0}+\nu$ has compact resolvent. In particular, there exists a positive weak solution to $\left(H_{0}+\nu\right) \Phi=\lambda_{0} \Phi$ for $\lambda_{0}:=\inf \sigma\left(H_{0}+\nu\right)$. This solution is unique (up to a factor) and belongs to $L^{2}(X)$. If $H_{0}+\nu$ satisfies a Harnack principle, then $\lambda_{0}$ is the only value in $\mathbb{R}$ allowing for a positive weak solution.

We can now state our result in the case of non-compact $X$.

Theorem 4.7. Let $(\mathcal{E}, \mathcal{D})$ be a regular, strictly local, irreducible Dirichlet form, $H_{0}$ be the associated operator and $\nu$ with $\nu_{+} \in \mathcal{M}_{R, 0}, \nu_{-} \in \mathcal{M}_{R, 1}$. Suppose that $\mathcal{E}$ satisfies the local compactness property and $X$ is noncompact. Then, if $\lambda<\inf \sigma\left(H_{0}+\nu\right)$ and $H_{0}+\nu$ satisfies the Harnack principle for $\lambda$, there is an a.e. positive solution of $\left(H_{0}+\nu\right) \Phi=\lambda \Phi$.

That we have to assume that $X$ is noncompact can easily be seen by looking at the Laplacian on a compact manifold. In that situation any positive weak solution must in fact be in $L^{2}$ due to the Harnack principle. Thus the corresponding energy must lie in the spectrum (see Theorem 4.6).

Characterizing the infimum of the spectrum. The previous results to not yet settle the existence of a positive weak solution for the groundstate energy inf $\sigma\left(H_{0}+\nu\right)$ in the noncompact case. The uniform Harnack principle settles this question: 
Theorem 4.8. Let $(\mathcal{E}, \mathcal{D})$ be a regular, strictly local, irreducible Dirichlet form, $H_{0}$ be the associated operator, $\nu$ with $\nu_{+} \in \mathcal{M}_{R, 0}, \nu_{-} \in \mathcal{M}_{R, 1}$. Suppose that $\mathcal{E}$ satisfies the local compactness property and $H_{0}+\nu$ satisfies the uniform Harnack principle. Then there is an a.e. positive weak solution of $\left(H_{0}+\nu\right) \Phi=\lambda \Phi$ for $\lambda=\inf \sigma\left(H_{0}+\nu\right)$.

\section{WeAK SOlUtions AND SPECTRUM}

In this section we relate energies in the spectrum to energies for which (suitably bounded) weak solutions exist. The results are taken from [23. The final characterization relies on [22] as well.

A Weyl type criterion. We include the following criterion for completeness. It is taken from [66, Lemma 1.4.4, see also [30], Lemma 4.1 for the same result in a slightly different formulation.

Proposition 5.1. Let $h$ be a closed, semibounded form and $H$ the associated selfadjoint operator. Then the following assertions are equivalent:

(i) $\lambda \in \sigma(H)$.

(ii) There exists a sequence $\left(u_{n}\right)$ in $\mathcal{D}(h)$ with $\left\|u_{n}\right\| \rightarrow 1$ and

$$
\sup _{v \in \mathcal{D}(h),\|v\|_{h} \leq 1}\left|(h-\lambda)\left[u_{n}, v\right]\right| \rightarrow 0,
$$

for $n \rightarrow \infty$.

A Caccioppoli type inequality. In this section we prove a bound on the energy measure of a generalized eigenfunction on a set in terms of bounds on the eigenfunction on certain neighborhood of the set.

We need the following notation: For $E \subset X$ and $b>0$ we define the $b$-neighborhood of $E$ as

$$
B_{b}(E):=\{y \in X: \rho(y, E) \leq b\} .
$$

Theorem 5.2. Let $\mathcal{E}$ be a strictly local regular Dirichlet form. Let $\mu_{+} \in \mathcal{M}_{0}$ and $\mu_{-} \in \mathcal{M}_{1}$ be given. Let $\lambda_{0} \in \mathbb{R}$ and $b_{0}>0$ be given. Then, there exists $a C=C\left(b_{0}, \lambda_{0}, \mu_{-}\right)$such that for any generalized eigenfunction $u$ to an eigenvalue $\lambda \leq \lambda_{0}$ of $H_{0}+\mu$ the inequality

$$
\int_{E} d \Gamma(u) \leq \frac{C}{b^{2}} \int_{B_{b}(E)}|u|^{2} d m
$$

holds for any closed $E \subset X$ and any $0<b \leq b_{0}$.

Remark. For compact $E$ both sides in the above inequality are finite, for $E$ merely closed, one or both sides might be infinite. In any case, it suffices to prove the compact case since $\Gamma$ is a Radon measure.

The Caccioppoli inequality replaces the familiar commutator estimates that are used for Schrödinger operators.

A $\frac{1}{2}$ Shnol type result: How suitably bounded solutions force spectrum. In this section, we first present an abstract Shnol type result. Unfortunately, we have to start with a disclaimer. In 23 we messed up the reference to Shnol's original result (as do many other authors). In fact, [59] is the correct citation but there are two more papers with quite similar titles [60, 61] and [59] does not appear in MathSciNet. 
The latter article deals with Schrödinger operators on the half line and says that for spectrally almost every $\lambda \in \mathbb{R}$ the solution on the eigenvalue problem is bounded by const $x^{\frac{1}{2}+\varepsilon}$ as $x \rightarrow \infty$ and vice versa. By "the solution" we mean a solution with the prescribed boundary condition at 0 and such a solution always exists since we are dealing with ODE. In this section we show $\frac{1}{2}$ Shnol, even a little stronger: if a weak solution with suitable exponential bounds exist for a given energy, that energy is in the spectrum.

We need the following notation. For $E \in X$ and $b>0$ we define the inner $b$-collar of $E$ as

$$
C_{b}(E):=\left\{y \in E: \rho\left(y, E^{c}\right) \leq b\right\} .
$$

Theorem 5.3. Let $\mathcal{E}$ be a strictly local regular Dirichlet form. Let $\mu_{+} \in \mathcal{M}_{0}$ and $\mu_{-} \in \mathcal{M}_{1}$ be given. Let $\lambda \in \mathbb{R}$ with generalized eigenfunction $u$ be given. If there exists $b>0$ and a sequence $\left(E_{n}\right)$ of compact subsets of $X$ with

$$
\frac{\left\|u \chi_{C_{b}\left(E_{n}\right)}\right\|}{\left\|u \chi_{E_{n}}\right\|} \longrightarrow 0, n \longrightarrow 0
$$

then $\lambda$ belongs to $\sigma(H)$.

We will now specialize our considerations to subexponentially bounded eigenfunctions.

A function $J:[0, \infty) \longrightarrow[0, \infty)$ is said to be subexponentially bounded if for any $\alpha>0$ there exists a $C_{\alpha} \geq 0$ with $J(r) \leq C_{\alpha} \exp (\alpha r)$ for all $r>0$. A $\mathbb{K}$-valued function $f$ on a pseudo metric space $(X, \rho)$ with measure $m$ is said to be subexponentially bounded if for some $x_{0} \in X$ and $\omega(x)=\rho\left(x_{0}, x\right)$ the function $e^{-\alpha \omega} u$ belongs to $L^{2}(X, m)$ for any $\alpha>0$. Recall that a strictly local regular Dirichlet form $\mathcal{E}$ gives rise to an intrinsic pseudo metric $\rho$ and an associated pseudo metric space $(X, \rho)$.

Theorem 5.4. Let $\mathcal{E}$ be a strictly local regular Dirichlet form, $x_{0} \in X$ arbitrary and $\omega(x)=\rho\left(x_{0}, x\right)$. Let $\mu_{+} \in \mathcal{M}_{0}$ and $\mu_{-} \in \mathcal{M}_{1}$ be given. Let $u \neq 0$ be a subexponentially bounded generalized eigenfunction. Then, $\lambda$ belongs to $\sigma(H)$.

A $\frac{1}{2}$ Shnol type result: How spectrum forces suitably bounded generalized eigenfunctions. In the last subsection we have discussed that existence of suitably bounded weak solutions implies that an energy belongs to the spectrum. In this section we discuss a converse given in 22] that was known before for ordinary Schrödinger operators; see the literature cited in the monograph [16].

Recall that $\mathcal{E}$ is called ultracontractive if for each $t>0$ the semigroup $e^{-t H_{0}}$ gives a map from $L^{2}(X)$ to $L^{\infty}(X)$.

Theorem 5.5. Let $\mathcal{E}$ be a strictly local, regular, ultracontractive Dirichlet form satisfying condition $(\mathrm{G})$. Let $\mu=\mu_{+}-\mu_{-}$with $\mu_{+} \in \mathcal{M}_{0}$ and $\mu_{-} \in \hat{\mathcal{S}}_{K}$ with $c_{\text {Kato }}(\mu)<1$. Define $H:=H_{0}+\mu$. Then for spectrally a.e. $\lambda \in \sigma(H)$ there is a subexponentially bounded generalized eigenfunction $u \neq 0$ with $H u=\lambda u$. 
Actually, as remarked in [22], one does arrive at generalized eigenfunctions with polynomial bounds if one assumes that the volume of balls grows polynomially as well.

A Shnol type result: Characterizing the spectrum by subexponentially bounded solutions. We can now put together the results of the preceeding subsections and obtain a characterization of the spectrum via subexponentially bounded solutions.

Corollary 5.6. Let $\mathcal{E}$ be a stricly local, regular, ultracontractive Dirichlet form satisfying $(\mathrm{G})$. Let $\mu=\mu_{+}-\mu_{-}$with $\mu_{+} \in \mathcal{M}_{0}$ and $\mu_{-} \in \hat{\mathcal{S}}_{K}$ with $c_{\text {Kato }}(\mu)<1$. Define $H:=H_{0}+\mu$. Then the spectral measures of $H$ are supported on

$$
\{\lambda \in \mathbb{R} \mid \exists \text { subexponentially bounded } u \neq 0 \text { with } H u=\lambda u\} .
$$

\section{EXAMPLES AND APPLICATIONS}

Several different types of operators to which our results can be applied have already been mentioned in Section 1. This includes classical examples like Schrödinger operators and symmetric elliptic second order differential operators on unbounded domains in $\mathbb{R}^{d}$. More generally, Laplace-Beltrami operators and rather general elliptic second order differential operators on Riemannian manifolds fall also within this class. In this section we will discuss in some more detail two types of examples which have attracted attention more recently, namely singular interaction Hamiltonians and quantum graphs. Moreover, we discuss here applications of the ground state transformation.

Hamiltonians with singular interactions. Hamiltonians with singular interactions arise when the Laplacian is perturbed by a perturbation which is localized on a set of Lebesgue measure zero. Here we consider more specifically operators with an interaction supported on an orientable, compact sub-manifold $M \subset \mathbb{R}^{d}$ of class $C^{2}$ and codimension one. The manifold $M$ may or may not have a boundary. In the sequel we follow roughly the exposition in [43]. For more background see [24] or Appendix K of [10].

The simplest type of Hamiltonian with a potential perturbation supported on $M$ is formally given by

$$
\left(H_{\alpha \sigma_{M}}\right) f(x):=(-\Delta-\alpha \cdot \delta(x-M)) f(x),
$$

where $\alpha>0$ is a coupling constant. To show that the operator $H_{\alpha \sigma_{M}}$ can be given a rigorous meaning we establish next that it falls into the framework outlined in Section 2 ,

For this purpose denote by $\nu_{M}$ the Dirac measure in $\mathbb{R}^{d}$ with support on $M$. This means that for any Borel set $G \subset \mathbb{R}^{d}$ we have $\nu_{M}(G)=$ $s_{d-1}(G \cap M)$. Here $s_{d-1}$ is the $d-1$ dimensional surface measure on $M$. From Theorem 4.1 in 24] we infer that the measure $\nu_{M}$ belongs to the Kato 
class. In particular, for such a measure and an arbitrary $a>0$ there exists $b_{a}<\infty$ such that

$$
\int_{\mathbb{R}^{d}}|\psi(x)|^{2} \nu_{M}(d x) \leq a\|\nabla \psi\|^{2}+b_{a}\|\psi\|^{2} .
$$

As mentioned in Section 2 this implies that the form $\mathcal{E}_{\alpha \nu_{M}}:=\mathcal{E}+\alpha \nu_{M}$ is closed on the domain $\mathcal{D}$ and densely defined. The unique selfadjoint operator associated to $\mathcal{E}_{\alpha \nu_{M}}$ acing on $L^{2}\left(\mathbb{R}^{d}\right)$ will be denoted by $H_{\alpha \nu_{M}}$.

It is possible to define the operator $H_{\alpha \nu_{M}}$ by appropriate selfadjoint boundary conditions on $M$, cf. [24, 43]. To explain this more precisely we need some notation. Denote by $\mathrm{n}: M \rightarrow \mathbb{S}^{d}$ a global unit normal vectorfield on $M$. Denote by $D\left(\tilde{H}_{\alpha \nu_{M}}\right)$ the set of functions

$$
\psi \in C\left(\mathbb{R}^{d}\right) \cap W^{1,2}\left(\mathbb{R}^{d}\right) \cap C^{\infty}\left(\mathbb{R}^{d} \backslash M\right) \cap W^{2,2}\left(\mathbb{R}^{d} \backslash M\right)
$$

which satisfy for all $x \in M$

$$
\lim _{\epsilon \searrow 0} \frac{\psi(x+\epsilon \mathrm{n}(x))-\psi(x)}{\epsilon}+\lim _{\epsilon \searrow 0} \frac{\psi(x-\epsilon \mathrm{n}(x))-\psi(x)}{\epsilon}=-\alpha \psi(x)
$$

Using Green's formula one concludes as in Remark 4.1 of [24] that the closure of $-\Delta$ with domain $D\left(\tilde{H}_{\alpha \nu_{M}}\right)$ is the selfadjoint operator $H_{\alpha \nu_{M}}$.

Since the measure $\nu_{M}$ belongs to the Kato class and is supported on a compact set, the essential spectrum of $H_{\alpha \nu_{M}}$ equals $[0, \infty)$, cf. Theorem 3.2 in [24]. In space dimension two $H_{\alpha \nu_{M}}$ has nonempty discrete spectrum for any positive value of the coupling constant $\alpha$. This can be seen using the proof of Corollary 11 in [25]. For higher dimensions there is a critical value $\alpha_{c}>0$ such that there exists a negative eigenvalue if and only if $\alpha \geq \alpha_{c}$, cf. the discussion on page 20 of [33].

Quantum graphs. Quantum graphs are given in terms of a metric graph $\Gamma$ and a Laplace (or more generally) Schrödinger operator $H$ defined on the edges of $\Gamma$ together with a set of (generalised) boundary conditions at the vertices which make $H$ selfadjoint. To make this more precise we define the geometric structure of metric graphs, as well as the operators acting on the associated $L^{2}$-Hilbert space.

We start with the definition of a metric graph which is appropriate for our purposes.

Definition 6.1. Let $V$ and $E$ be countable sets, $l_{-}$a positive real, and $\mathcal{G}$ a map

$$
\mathcal{G}: E \rightarrow V \times V \times\left[l_{-}, \infty\right], \quad e \mapsto\left(\iota(e), \tau(e), l_{e}\right) .
$$

Here $\left[l_{-}, \infty\right]$ means $\left[l_{-}, \infty\right) \cup\{+\infty\}$. We call the triple $\Gamma=(V, E, \mathcal{G})$ a metric graph, elements of $V=V(\Gamma)$ vertices, elements of $E=E(\Gamma)$ edges, $\iota(e)$ the initial vertex of $e, \tau(e)$ the terminal vertex of $e$ and $l_{e}$ the length of e. Both $\iota(e)$ and $\tau(e)$ are called endvertices of $e$, or incident to e. The number of edges incident to the vertex $v$ is called the degree of $v$. We assume that the degree is finite for all vertices.

Note that the two endvertices of an edge are allowed to coincide and there may be multiple edges connecting two vertices. We let $X_{e}:=\{e\} \times\left(0, l_{e}\right)$, $X=X_{\Gamma}=V \cup \bigcup_{e \in E} X_{e}$ and $\overline{X_{e}}:=\{e\} \times\left[0, l_{e}\right]$. On the set $X$ it is possible 
to define in a natural way the length of paths and, using this notion, also a metric, cf. Section 1 in 44 .

Now we introduce the relevant Hilbert spaces on which the Laplace, respectively, Schrödinger operators will act. For $k \in\{0,1,2\}$ we set

$$
W^{k, 2}(E):=\bigoplus_{e \in E} W^{k, 2}\left(0, l_{e}\right)
$$

and for $W^{0,2}(E)$ we use the usual notation $L^{2}(E)$. Given $k \in\{0,1,2\}$ and a function $u \in W^{1, k}(E)$ we denote by $u_{e}$ the projection of $u$ to the space $W^{k, 2}\left(0, l_{e}\right)$. Thus we can identify each $u \in W^{1, k}(E)$ with a family $\left(u_{e}\right)_{e \in E}, u_{e} \in W^{0,2}\left(0, l_{e}\right)$.

Next we discuss pointwise properties of functions in $u \in W^{1, k}\left(0, l_{e}\right)$. Recall that for any $l>0$ any element $h$ of $W^{1,2}(0, l)$ has a continuous version; we will always pick this version and then the boundary value $h(0):=\lim _{x \rightarrow 0+} h(x)$ exists and satisfies

$$
|h(0)|^{2} \leq \frac{2}{l}\|h\|_{L^{2}(0, l)}^{2}+l\left\|h^{\prime}\right\|_{L^{2}(0, l)}^{2}
$$

by standard Sobolev type theorems. Consider now an edge $e$, the vertex $v=\iota(e) \in V$ and $u \in W^{1,2}\left(0, l_{e}\right)$. Then the limit $u(v):=\lim _{t \rightarrow 0} u(t)$ exists, as well as $u(w):=\lim _{t \rightarrow l_{e}} u(t)$ for $w=\tau(e)$ and (6.2) holds (with the obvious modifications). Similarly, for an edge $e$ and the vertex $v=\iota(e)$ and the vertex $w=\tau(e)$ and $u \in W^{2,2}\left(0, l_{e}\right)$ the limits $u^{\prime}(v):=\lim _{x \rightarrow v, x \in e} u^{\prime}(x)$ and $u^{\prime}(w):=-\lim _{x \rightarrow w, x \in e} u^{\prime}(x)$ exist. Note that our sign convention is such that the definition of the derivative is canonical, i. e. independent of the choice of orientation of the edge. For $f \in W^{1,2}(E)$ and each vertex $v$ we gather the boundary values of $f_{e}(v)$ over all edges $e$ adjacent to $v$ in a vector $f(v)$. More precisely, denote by $E_{v}:=\{e \in E \mid v \in\{\iota(e), \tau(e)\}\}$ the set of vertices adjacent to $v$ and define $f(v):=\left(f_{e}(v)\right)_{e \in E_{v}} \in \mathbb{C}^{E_{v}}$ and similarly, for $f \in W^{2,2}(E)$ we further collect the boundary values of $f_{e}^{\prime}(v)$ over all edges $e$ adjacent to $v$ in a vector $f^{\prime}(v) \in \mathbb{C}^{E_{v}}$. These boundary values of functions will be used to define the boundary conditions of the Laplacian, respectively the domains of definition of the forms we will be considering. Here we restrict ourselves to Kirchhoff boundary conditions and call a function $\left(u_{e}\right)_{e \in E} \in W^{1,2}(E)$ continuous, if, for any vertex $v$ and all edges adjacent to it $u_{e}(v)=u_{e^{\prime}}(v)$. Now set

$$
\begin{aligned}
D\left(s_{0}\right) & :=W^{1,2}(E) \cap C(X) \\
s_{0}(f, g) & :=\sum_{e \in E} \int_{0}^{l(e)} f_{e}^{\prime}(t) \bar{g}_{e}^{\prime}(t) d t
\end{aligned}
$$

Obviously, the form $s_{0}$ is bounded below, closed a Dirichlet form and strongly local. Hence, there exists a unique associated self-adjoint operator which we denote by $H_{P}$. It can be explicitly characterized by

$$
\begin{aligned}
D\left(H_{K}\right) & :=\left\{f \in W^{2,2}(E) \cap C(X): \sum_{e \in E_{v}} f_{e}(v)=0 \text { for all } v \in V\right\} \\
\left(H_{K} f\right)_{e}: & =-f_{e}^{\prime \prime} \text { for all } e \in E .
\end{aligned}
$$

It is possible to define quantum graphs with more general generalised boundary conditions at the vertices but not all reasonable choices will lead to 
Dirichlet forms; in [40] a characterization of those boundary conditions for which the form is a Dirichlet form is given. However the setup is somewhat different from ours.

Applications. The ground state transformation which featured in Theorem 4.1 can be used to obtain a formula for the lowest spectral gap. To be more precise let us assume that $\mathcal{E}, \nu$ and $\Phi$ satisfy the conditions of Theorem 4.1. Assume in addition that $\Phi$ is in $\mathcal{D}(\mathcal{E}+\nu)$. Then $\Phi$ is an eigenfunction of $H$ corresponding to the eigenvalue $\lambda=\min \sigma(H)$. We denote by

$$
\lambda^{\prime}:=\inf \{\mathcal{E}[u, u]+\nu[u, u] \mid u \in \mathcal{D},\|u\|=1, u \perp \Phi\}
$$

the second lowest eigenvalue below the essential spectrum of $H$, or, if it does not exist, the bottom of $\sigma_{e s s}(H)$. Then we obtain the following formula

$$
\lambda^{\prime}-\lambda=\inf _{\{u \in \mathcal{D}(\mathcal{E}+\nu),\|u\|=1, u \perp \Phi\}} \int_{X} \Phi^{2} d \Gamma\left(u \Phi^{-1}, u \Phi^{-1}\right)
$$

which determines the lowest spectral gap. It has been used in [42, 43, 72] to derive lower bounds on the distance between the two lowest eigenvalues of different classes of Schrödinger operators (see [63] for a related approach). In [42] bounded potentials are considered, in [43] singular interactions along curves in $\mathbb{R}^{2}$ are studied, and 72 generalizes these results using a unified approach based on Kato-class measures.

If for a subset $U \subset X$ of positive measure and a function $u \in \mathcal{D}$ with $\|u\|=1$ and $u \perp \Phi\}$ the non-negative measure $\Gamma\left(u \Phi^{-1}, u \Phi^{-1}\right)$ is absolutely continuous with respect to $m$, one can exploit formula (6.5) to derive the following estimate (cf. Section 3 in [72, and [42, 43] for similar bounds). Denote by $\gamma\left(u \Phi^{-1}\right)=\frac{d \Gamma\left(u \Phi^{-1}, u \Phi^{-1}\right)}{d m}$ the Radon-Nykodim derivative. Then an application of the Cauchy-Schwarz inequality gives

$$
\int_{U} \Phi^{2} d \Gamma\left(u \Phi^{-1}, u \Phi^{-1}\right) \geq \frac{1}{m(U)} \inf _{U} \Phi^{2}\left(\int_{U} \sqrt{\gamma\left(u \Phi^{-1}\right)} d m\right)^{2}
$$

Now we formulate more precisely the setting in which the above mentioned results [42, 43, 72] apply. In fact, we choose here to formulate the main theorem of [72]. It applies to more general situations than [42] and [43] and is formulated in the language of Dirichlet forms. Consider the case where $X=\mathbb{R}^{d}, \mathcal{E}$ is equal to the classical Dirichlet form, $\nu$ is a non-negative, compactly supported measure satisfying for some $c_{\nu} \in(0, \infty), \alpha \in[0,2)$ the bound $\nu(B(x, r)) \leq c_{\nu} r^{d-\alpha}$ for all $r>0, x \in \mathbb{R}^{d}$, and $D$ denotes the diameter of the support of $\nu$. Let us assume that the bottom of the spectrum of $\mathcal{E}+\nu$ consists of two isolated eigenvalues, which will be denoted by $\lambda_{0}<\lambda_{1}$. Under these assumptions there exist constants $C, C_{0}, p, q \in(0, \infty)$ such that

$$
\lambda_{1}-\lambda_{0} \geq \frac{C}{\left(c_{\nu}+1\right)^{p}(D+1)^{q}} \cdot\left|\lambda_{0}\right| \cdot e^{-C_{0}(D+1) \cdot \sqrt{\left|\lambda_{0}\right|}}
$$

The ground state transformation plays an important role in other situations as well. It is for instance used in the the study of $L^{p}-L^{q}$ mapping properties of the semigroup associated to $\mathcal{E}$ [29]. In the theory of random Schrödinger operators it is used to remove a symmetry condition from the proof of of Lifschitz tails [48. 


\section{REFERENCES}

[1] S. Agmon. Lower bounds for solutions of Schrödinger equations. J. Anal. Math., 23:1-25, 1970.

[2] S. Agmon. On Positive Solutions of Elliptic Equations with Periodic Coefficients in $R^{N}$, Spectral Results and Extensions to Elliptic Operators on Riemannian Manifolds in Differential Equations North Holland, Amsterdam, 1984.

[3] S. Agmon and L. Hörmander. Asymptotic properties of solutions of differential equations with simple characteristics. J. d'Analyse Math., 30:1-38, 1976.

[4] S. Agmon. Lectures on elliptic boundary value problems. Van Nostrand Mathematical Studies, No. 2. Van Nostrand, Princeton, 1965.

[5] S. Agmon. Lectures on exponential decay of solutions of second-order elliptic equations: bounds on eigenfunctions of $N$-body Schrödinger operators. Princeton University Press, Princeton, N.J., 1982.

[6] S. Agmon. Bounds on exponential decay of eigenfunctions of Schrödinger operators. In Schrödinger operators (Como, 1984), volume 1159 of Lecture Notes in Math., pages 1-38. Springer, Berlin, 1985.

[7] S. Agmon. On positivity and decay of solutions of second order elliptic equations on Riemannian manifolds. In Methods of functional analysis and theory of elliptic equations (Naples, 1982), pages 19-52. Liguori, Naples, 1983.

[8] M. Aizenman and B. Simon. Brownian motion and Harnack inequality for Schrödinger operators. Commun. Pure Appl. Math., 35:209-273, 1982.

[9] M. Aizenman, R. Sims, and S. Warzel. Absolutely continuous spectra of quantum tree graphs with weak disorder. Comm. Math. Phys., 264(2):371-389, 2006.

[10] S. Albeverio, F. Gesztesy, R. Høegh-Krohn, and H. Holden. Solvable models in quantum mechanics. AMS Chelsea Publishing, Providence, RI, second edition, 2005. With an appendix by P. Exner.

[11] S. Albeverio and Z. M. Ma. Perturbation of Dirichlet forms - lower semiboundedness, closability, and form cores. J. Funct. Anal., 99(2):332-356, 1991.

[12] W. Allegretto. On the equivalence of two types of oscillation for elliptic operators. Pac. J. Math., 55:319-328, 1974.

[13] W. Allegretto. Spectral estimates and oscillation of singular differential operators. Proc. Am. Math. Soc., 73:51, 1979.

[14] W. Allegretto. Positive solutions and spectral properties of second order elliptic operators. Pac. J. Math., 92:15-25, 1981.

[15] C.M. Bender, S. Boettcher and P. Meisinger. PT symmetric quantum mechanics. J.Math.Phys., 40:2201-2229, 1999.

[16] Ju. M.Berezanskii. Expansions in eigenfunctions of selfadjoint operators. Translated from the Russian by R. Bolstein, J. M. Danskin, J. Rovnyak and L. Shulman. Translations of Mathematical Monographs, Vol. 17 American Mathematical Society, Providence, R.I. 1968

[17] A. Beurling and J. Deny. Dirichlet spaces. Proc. Nat. Acad. Sci. U.S.A., 45:208-215, 1959.

[18] M. Biroli. Schrödinger type and relaxed Dirichlet problems for the subelliptic $p$ Laplacian. Potential Analysis, 15, 1-16, 2001.

[19] M. Biroli and S. Marchi. Harnack inequality for the Schrödinger problem relative to strongly local Riemannian $p$-homogeneous forms with a potential in the Kato class. Bound. Value Probl. 2007, Art. ID 24806, 19 pp.

[20] M. Biroli and U. Mosco. A Saint-Venant type principle for Dirichlet forms on discontinuous media. Ann. Mat. Pura Appl., IV. Ser., 169:125-181, 1995.

[21] N. Bouleau and F. Hirsch. Dirichlet forms and analysis on Wiener space, volume 14 of de Gruyter Studies in Mathematics. Walter de Gruyter \& Co., Berlin, 1991.

[22] A. Boutet de Monvel and P. Stollmann. Eigenfunction expansions for generators of Dirichlet forms. J. Reine Angew. Math., 561:131-144, 2003. 
[23] A. Boutet de Monvel, D. Lenz, and P. Stollmann. Schnol's theorem for strongly local forms. Israel J. Math., to appear, 2008.

[24] J. F. Brasche, P. Exner, Y. A. Kuperin, and P. Šeba. Schrödinger operators with singular interactions. J. Math. Anal. Appl., 184(1):112-139, 1994.

[25] J. F. Brasche. On eigenvalues and eigensolutions of the Schrödinger equation on the complement of a set with classical capacity zero Methods Funct. Anal. Topology, 9(3), 189-206, 2003. http://www.math.chalmers.se/ brasche/ett.pdf

[26] F. Chiarenza, E. Fabes, and N. Garofalo. Harnack's inequality for Schrödinger operators and the continuity of solutions. Proc. Amer. Math. Soc., 98(3):415-425, 1986.

[27] H. L. Cycon, R. G. Froese, W. Kirsch, and B. Simon. Schrödinger Operators with Application to Quantum Mechanics and Global Geometry. Text and Monographs in Physics. Springer, Berlin, 1987.

[28] E. B. Davies. Spectral theory and differential operators. Cambridge University Press, Cambridge, 1995.

[29] E. B. Davies and B. Simon. Ultracontractivity and the heat kernel for Schrödinger operators and Dirichlet Laplacians. J. Funct. Anal., 59(2):335-395, 1984.

[30] Y. Dermenjian, M. Durand and V. Iftimie: Spectral analysis of an acoustic multistratified perturbed cylinder. Comm. Partial Differential Equations, 23 (1-2): 141-169 (1998)

[31] A. F. M. ter Elst and D. W. Robinson: Invariant subspaces of submarkovian semigroups. J. Evol. Equ., 8 (4): 661-671 (2008)

[32] P. Exner. An isoperimetric problem for leaky loops and related mean-chord inequalities. J. Math. Phys., 46(6):062105, 2005. http://arxiv.org/abs/math-ph/0501066

[33] P. Exner and K. Yoshitomi. Eigenvalue asymptotics for the SchrA 9 dinger operator with a $\delta$-interaction on a punctured surface. Lett. Math. Phys., 65(1): 19-26 (2003).

[34] R. Frank, D. Lenz, D. Wingert. Intrinsic metrics for non-local symmetric Dirichlet forms and applications. in preparation.

[35] M. Fukushima. Dirichlet forms and Markov processes. North-Holland Mathematical Library, Vol. 23. Amsterdam - Oxford -New York: North-Holland Publishing Company. Tokyo: Kodansha Ltd. X, 196 p., 1980.

[36] M. Fukushima, Y. Oshima, and M. Takeda. Dirichlet forms and symmetric Markov processes. de Gruyter Studies in Mathematics. 19. Berlin: Walter de Gruyter. viii, 392 p., 1994.

[37] W. Hansen. Harnack inequalities for Schrödinger operators. Ann. Scuola Norm. Sup. Pisa Cl. Sci. (4), 28(3):413-470, 1999.

[38] I. W. Herbst and A. D. Sloan. Perturbation of translation invariant positivity preserving semigroups on $L^{2}\left(\mathbf{R}^{N}\right)$. Trans. Amer. Math. Soc., 236:325-360, 1978.

[39] M. Hoffmann-Ostenhof, T. Hoffmann-Ostenhof, and N. Nadirashvili. Interior Hölder estimates for solutions of Schrödinger equations and the regularity of nodal sets. Commun. Part. Diff. Eqns, 20:1241-1273, 1995.

[40] U. Kant, T. Klauß, J. Voigt and M. Weber. Dirichlet forms for singular onedimensional operators and on graphs. J. Evol. Equ., to appear.

[41] M. Kassmann. Harnack inequalities: An Introduction. Bound. Value Probl. 2007, Art. ID 81415,21 pp. 35-01

[42] W. Kirsch and B. Simon. Comparison theorems for the gap of Schrödinger operators. J. Funct. Anal., 75:396-410, 1987.

[43] S. Kondej and I. Veselić. Lower bounds on the lowest spectral gap of singular potential Hamiltonians. Ann. Henri Poincaré, 8(1):109-134, 2006.

[44] D. Lenz, C. Schubert, P. Stollmann. Eigenfunction expansion for Schrodinger operators on metric graphs. Integral Equations and Operator Theory 62 (2008), 541-553.

[45] D. Lenz, P. Stollmann and I. Veselić. The Allegretto-Piepenbrinck Theorem for strongly local Dirichlet forms. : Documenta Mathematica, 14:167-190 (2009).

[46] D. Lenz, P. Stollmann and I. Veselić. Irreducibility and connectedness for Dirichlet forms. In preparation, 2009

[47] Z.-M. Ma and M. Röckner. Introduction to the theory of (non-symmetric) Dirichlet forms. Universitext. Berlin: Springer-Verlag, 1992. 
[48] G. A. Mezincescu. Lifschitz singularities for periodic operators plus random potentials. J. Statist. Phys., 49(5-6):1181-1190, 1987.

[49] J. Moser. On harnacks theorem for elliptic differential equations. Comm. Pure Appl. Math., 14:577-591, 1961.

[50] W.F. Moss and J. Piepenbrink. Positive solutions of elliptic equations. Pacific J. Math., 75(1):219-226, 1978.

[51] J. Piepenbrink. Nonoscillatory elliptic equations. J. Differential Equations, 15:541$550,1974$.

[52] J. Piepenbrink. A conjecture of Glazman. J. Differential Equations, 24(2):173-177, 1977.

[53] Y. Pinchover. Topics in the theory of positive solutions of second-order elliptic and parabolic partial differential equations. In Spectral theory and mathematical physics: a Festschrift in honor of Barry Simon's 60th birthday, volume 76 of Proc. Sympos. Pure Math., pages 329-355. Amer. Math. Soc., Providence, RI, 2007.

[54] R.G. Pinsky. Positive harmonic functions and diffusion, volume 45 of Cambridge Studies in Advanced Mathematics. Cambridge University Press, Cambridge, 1995.

[55] M. Reed and B. Simon. Methods of Modern Mathematical Physics II, Fourier Analysis, Self-Adjointness. Academic Press, San Diego, 1975.

[56] M. Reed and B. Simon. Methods of Modern Mathematical Physics IV, Analysis of Operators. Academic Press, San Diego, 1978.

[57] L. Saloff-Coste. Parabolic Harnack inequality for divergence-form second-order differential operators. Potential Anal., 4(4):429-467, 1995. Potential theory and degenerate partial differential operators (Parma).

[58] J. Serrin. Local behavior of solutions of quasi-linear equations. Acta Math., 111:247302, 1964.

[59] È. È. Shnol. On the behaviour of eigenfunctions. (Russian) Dokl. Akad. Nauk SSSR, n. Ser. 94, 389-392 (1954).

[60] È. È. Shnol. The behaviour of eigenfunctions and the spectrum of Sturm-Liouville operators. (Russian) Usp. Mat. Nauk 9, No.4(62), 113-132 (1954)

[61] È. È. Shnol. On the behavior of the eigenfunctions of Schrödinger's equation. (Russian) Mat. Sb. (N.S.) 42 (84) (1957), 273-286; erratum 46 (88) 1957259.

[62] B. Simon. Schrödinger semigroups. Bull. Amer. Math. Soc. 7 (3): 447-526 (1982).

[63] I. M. Singer, B. Wong, S.-T. Yau, and S. S.-T. Yau. An estimate of the gap of the first two eigenvalues in the Schrödinger operator. Ann. Scuola Norm. Sup. Pisa Cl. Sci. (4), 12(2):319-333, 1985.

[64] P. Stollmann. Smooth perturbations of regular Dirichlet forms. Proc. Am. Math. Soc., 116(3):747-752, 1992.

[65] P. Stollmann and J. Voigt. Perturbation of Dirichlet forms by measures. Potential Anal., 5(2):109-138, 1996.

[66] P. Stollmann. Caught by disorder: A Course on Bound States in Random Media, volume 20 of Progress in Mathematical Physics. Birkhäuser, 2001.

[67] K.-T. Sturm. Analysis on local Dirichlet spaces. I: Recurrence, conservativeness and $L^{p}$-Liouville properties.

[68] K.-T. Sturm. Measures charging no polar sets and additive functionals of Brownian motion. Forum Math., 4(3):257-297, 1992.

[69] K.-T. Sturm. Harnack's inequality for parabolic operators with singular low order terms. Math. Z., 216(4):593-611, 1994.

[70] K.T. Sturm. Analysis on local Dirichlet spaces. III: The parabolic Harnack inequality. J. Math. Pures Appl. (9) 75 (1996), 273-297.

[71] D. Sullivan. Related aspects of positivity in Riemannian geometry. J. Differential Geom., 25(3):327-351, 1987.

[72] H. Vogt. A lower bound on the first spectral gap of Schrödinger operators with Kato class measures. Ann. Henri Poincaré, 10(2):395-414, (2009) 
1 Mathematisches Institut, Friedrich-Schiller Universität Jena, ErnstAbbé Platz 2, 07743 Jena, Germany

E-mail address: daniel.lenz@uni-jena.de

$U R L:$ http://www.analysis-lenz.uni-jena.de/

${ }^{2}$ Fakultät für Mathematik, Technische Universität, 09107 Chemnitz, GerMANY

E-mail address: peter.stollmann@mathematik.tu-chemnitz.de

${ }^{3}$ Emmy-Noether-Project Schrödingeroperatoren, FAKultät FÜR MAThematik, 09107 TU Chemnitz, Germany

URL: http://www.tu-chemnitz.de/mathematik/enp/ 